\title{
Sleeve gastrectomy rapidly enhances islet function independently of body weight
}

\author{
Jonathan D. Douros, ${ }^{1}$ Jingjing Niu, ${ }^{1}$ Sophia Sdao, ${ }^{2}$ Trillian Gregg, ${ }^{2}$ Kelsey Fisher-Wellman, ${ }^{1}$ \\ Manish Bharadwaj, ${ }^{3}$ Anthony Molina, ${ }^{3}$ Ramamani Arumugam, ${ }^{1}$ MacKenzie Martin, ${ }^{1}$ \\ Enrico Petretto, ${ }^{4}$ Matthew J. Merrins, ${ }^{2}$ Mark A. Herman, ${ }^{1}$ Jenny Tong, ${ }^{1}$ Jonathan Campbell, ${ }^{1}$ \\ and David D'Alessio' \\ 'Division of Endocrinology, Duke Molecular Physiology Institute, Duke University, Durham, North Carolina, USA. \\ 2Department of Medicine, Division of Endocrinology, Diabetes, and Metabolism, University of Wisconsin-Madison, \\ Madison, Wisconsin, USA. ${ }^{3}$ Center for Diabetes Research, Wake Forest School of Medicine, Winston-Salem, North \\ Carolina, USA. ${ }^{4}$ Centre for Computational Biology, Duke-NUS Medical School, Singapore.
}

Bariatric surgeries including vertical sleeve gastrectomy (VSG) ameliorate obesity and diabetes. Weight loss and accompanying increases to insulin sensitivity contribute to improved glycemia after surgery; however, studies in humans also suggest weight-independent actions of bariatric procedures to lower blood glucose, possibly by improving insulin secretion. To evaluate this hypothesis, we compared VSG-operated mice with pair-fed, sham-surgical controls (PF-Sham) 2 weeks after surgery. This paradigm yielded similar postoperative body weight and insulin sensitivity between VSG and calorically restricted PF-Sham animals. However, VSG improved glucose tolerance and markedly enhanced insulin secretion during oral nutrient and i.p. glucose challenges compared with controls. Islets from VSC mice displayed a unique transcriptional signature enriched for genes involved in $\mathrm{Ca}^{2+}$ signaling and insulin secretion pathways. This finding suggests that bariatric surgery leads to intrinsic changes within the islet that alter function. Indeed, islets isolated from VSG mice had increased glucose-stimulated insulin secretion and a left-shifted glucose sensitivity curve compared with islets from PF-Sham mice. Isolated islets from VSC animals showed corresponding increases in the pulse duration of glucose-stimulated $\mathrm{Ca}^{2+}$ oscillations. Together, these findings demonstrate a weight-independent improvement in glycemic control following VSG, which is, in part, driven by improved insulin secretion and associated with substantial changes in islet gene expression. These results support a model in which $\beta$ cells play a key role in the adaptation to bariatric surgery and the improved glucose tolerance that is typical of these procedures.

Conflict of interest: The authors have declared that no conflict of interest exists.

Copyright: (c) 2019 American Society for Clinical Investigation

Submitted: December 7, 2018 Accepted: February 11, 2019 Published: March 21, 2019

\section{Reference information:} JCI Insight. 2019;4(6):e126688 https://doi.org/10.1172/jici. insight.126688.

\section{Introduction}

Bariatric surgery represents an effective treatment for weight loss and also has dramatic effects on diabetes, causing protracted remission in $40 \%-50 \%$ of affected patients $(1,2)$. There are a number of common surgical approaches, each of which makes distinctive modifications to the gastrointestinal anatomy and may play a role in improving glucose control. Vertical sleeve gastrectomy (VSG), now the most common bariatric procedure in the US (2), removes a large percentage of the body of the stomach, converting this distensible muscular organ into a tight sleeve. Roux-en Y gastric bypass (RYGB) reduces the stomach to a small pouch that empties directly into the upper jejunum and diverts biliopancreatic secretions to the distal small intestine. Adjustable gastric banding attaches an adjustable band to the proximal end of the stomach, restricting entry of food to a small pouch. The RYGB and VSG dramatically increase gastric-emptying rates (3-5), modify nutrient absorption (6-9), alter gastric emptying rates, and enhance incretin secretion (10). All of these factors may play a role in the contemporaneous reductions in body weight (BW) and insulin resistance, which contribute to improved glucose control (11-13) - particularly at later time points following surgery. However, numerous studies show that postsurgical improvements in glycemia occur prior to significant weight loss (14-16) and are superior to those seen in with weight loss alone (16). Thus, the full complement of mechanisms responsible for improved glucose regulation after bariatric surgery remain unknown, despite intensive investigation $(17,18)$. 
In the first weeks to months following surgery, patients with diabetes demonstrate some restoration of the acute insulin response to intravenous (i.v.) glucose (19-21), the loss of which is a hallmark feature of diabetic islet dysfunction (22). The early return of the acute insulin response is weight independent and is not directly dependent on insulinotropic factors from the surgically modified gut since the $\beta$ cell stimulus is given i.v. This observation suggests that bariatric surgery enhances islet function in a manner that does not require acute input from neuroendocrine sources. The studies described herein test the hypothesis that VSG improves glucose tolerance by enhancing insulin secretion. In these studies, we compare mice with VSG to pair-fed, sham-surgical controls (PF-Sham) to assess insulin secretion in vivo and ex vivo independently of changes in body weight, insulin demand, or caloric balance.

\section{Results}

Preoperative metabolic parameters in high-fat diet-fed mice. Presurgical BW for a representative cohort of mice on high-fat diet (HFD; Research Diets, catalog D12451, 45\% kcal from lipid; $n=15$ ) over 8 weeks did not differ between animals before either VSG or sham surgery (Supplemental Figure 1A; supplemental material available online with this article; https://doi.org/10.1172/jci.insight.126688DS1). Animals achieved a preoperative BW of $36.5 \pm 1.07 \mathrm{~g}$, with $\sim 31 \%$ of the weight gain occurring in the last week before surgery. We speculate that this may be due to the Ensure meal transition 2 days before surgery or to accumulated metabolic stress during HFD exposure. Neither glucose excursion nor circulating insulin concentrations differed between groups allocated to VSG or Sham surgery during preoperative i.p. glucose tolerance tests (IPGTTs) or mixed-meal tolerance tests (MMTTs; Supplemental Figure 1, B-E).

In vivo insulin secretion is enhanced by VSG during enteral nutrient delivery. A schematic of the experimental paradigm employed is shown in Figure 1A. During the first 2 weeks following surgery, both groups lost $\sim 18 \%$ BW (7.46 $\pm 0.62 \mathrm{~g}$; Figure 1B). BW and blood glucose during insulin tolerance tests (shown as percentage of baseline glucose; Figure 1C) did not differ between groups, indicating comparable insulin sensitivity between the 2 groups. However, 6-hour fasting blood glucose was significantly decreased in the VSG group by $\sim 13 \%$ (19 mg/dl; Figure 1D). Blood glucose during the MMTT peaked higher in VSG animals than controls 10 minutes following Ensure gavage, with a more rapid decline to baseline levels within 30 minutes (Figure 1E). While the glycemic profile differed in the controls, the integrated glucose AUC after Ensure gavage was similar between groups (Figure 1E). Fasting levels of insulin were similar in VSG and PF-Sham mice, but the postprandial rise was significantly greater in the VSG group 10 minutes after meal administration (Figure 1F). Plasma GLP-1 and GIP and were also elevated in the VSG group at 10 minutes, while glucagon was increased at 0 and 10 minutes (Figure 1, G-I). Following an overnight fast and subsequent 30-minute refeeding period, blood glucose was comparable between groups, but insulin levels were significantly elevated in the mice with VSG (Figure 1, J and K). Plasma insulin, total GLP-1, GIP, and glucagon levels were also significantly elevated in the VSG group compared with PF-Shams after refeeding (Figure 1, L-N). Taken together, these findings demonstrate significantly greater meal-stimulated insulin secretion in animals with VSG. The concurrent increase in incretins in the VSG group supports their role in at least some of the heightened $\beta$ cell response to meals, similar to what has been described in humans with this operation $(10,23)$.

Glucose stimulated insulin secretion (GSIS) is elevated after VSG. To test $\beta$ cell function independently of incretin stimulation, VSG and Sham mice were given IPGTT, in which glucose bypasses the surgically modified gut. Glycemic excursion was significantly reduced in VSG animals during an IPGTT (integrated glucose AUC reduced 33\%; Figure 2A). Additionally, plasma insulin 10 minutes after the i.p. glucose administration, and the insulin/glucose ratio, were both elevated in the VSG group $(\sim 47 \%$ and $\sim 63 \%$, respectively; Figure 2, B and C). Insulin secretion $(\mathrm{ng} / \mathrm{ml})$ and glucose excursion $\left(\mathrm{AUC}_{\mathrm{IPGTT}}\right)$ were inversely correlated during the IPGTT in the VSG group $\left(\mathrm{r}^{2}=0.21, P \leq 0.01\right.$; Figure 2D), but not the PF-Shams. These results suggest 2 important points. First, $\beta$ cells demonstrate greater sensitivity to glucose that is in part independent of acute stimulation by gut-derived factors after VSG. Second, enhanced insulin secretion contributes to the improved glucose tolerance seen with VSG.

VSG significantly modifies the islet transcriptome, including pathways linked to insulin secretion. Because VSG enhanced both glucose clearance and insulin secretion after an IPGTT, we hypothesized that there were adaptive changes within the islet in response to surgery, some of which might be transcriptionally mediated. This hypothesis was supported by RNA sequencing (RNA-seq) analysis of islets obtained from VSG and control mice. Within 2 weeks of surgery, VSG imparted a distinct transcriptional 

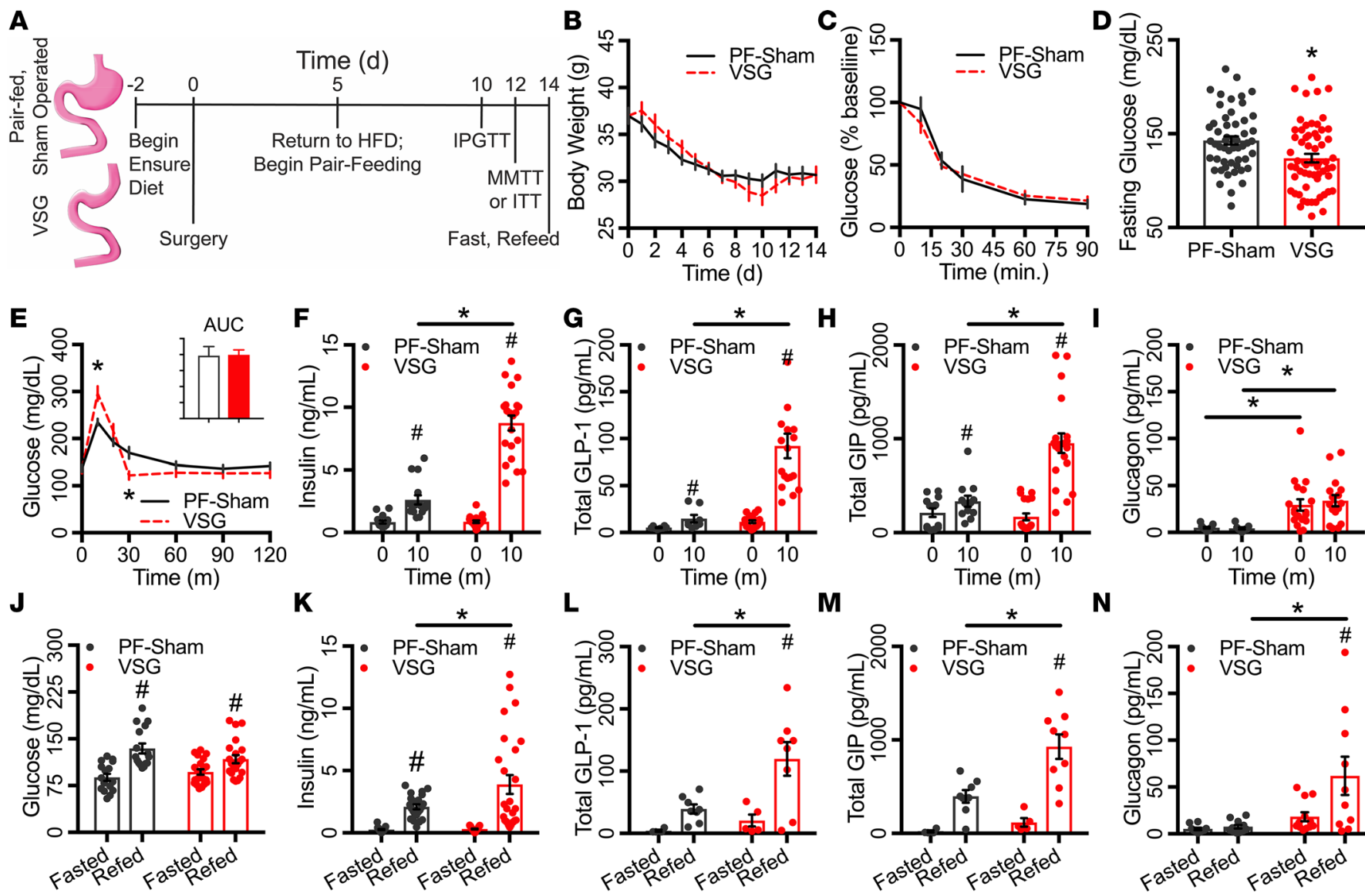

Figure 1. VSG rapidly improved glucose control and in vivo insulin secretion during enteral nutrient delivery independent of body weight. (A) Schematic of the experimental paradigm employed. (B) Body weight for 2 weeks following surgery (PF-Sham, $n=15$; VSG, $n=20$ ). (C and D) Blood glucose during insulin tolerance test (percentage of baseline glucose; PF-Sham, $n=15$; VSG, $n=20$ ) (C) and following 6-hour fast (PF-Sham, $n=53$; VSG, $n=60$ ) (D). (E-I) Blood glucose and integrated AUC during a mixed meal tolerance test (E), along with circulating insulin (F), total GLP-1 (G), GIP (H), and glucagon (I) at 0 minutes and 10 minutes after ensure gavage (PF-Sham, $n=7-16$; VSG, $n=19-21)$. (J-N) Blood glucose (J), and circulating insulin (K) following an overnight fast/30-minute refeed in PF-Sham ( $n=16-22)$ and VSG mice $(n=18-26)$, along with corresponding fasted and refed total GLP-1 (L), GIP (M), and glucagon levels (N) (PF-Sham, $n=4-12$; VSG, $n=4-11$ ). PF-Sham animals are shown with black solid lines or gray circles; VSG animals are shown in red dashed lines or red circles. Data represent mean \pm SEM; ${ }^{*} P<0.05$ between PF-Sham and VSG groups; ${ }^{*} P<0.05$ between time points within groups.

signature in islets. Volcano plots illustrate 611 significantly differentially expressed genes (DEG) between VSG and PF-Sham (Figure 3A); 459 between VSG and ad libitum-fed, sham-operated group (AL-Sham; Figure 3B); and 38 between PF-Sham and AL-Sham (Figure 3C) islets (Bonferroni adjusted $P<0.05$; for DEG list, see Supplemental Table 1). Hierarchical clustering of the samples based on the expression levels of the 100 most variable genes showed a distinct grouping of the VSG group apart from the AL-Sham and PF-Sham groups (Figure 3D). Hierarchical clustering also appeared to distinguish the majority of AL-Sham from PF-Sham mice. Principal component analysis (PCA) of the transcriptional data showed statistically significant separation of VSG samples away from the AL- and PF-Sham samples along the axis of the first principal component; these separations accounted for $73 \%$ of the variance in islet gene expression levels (Figure 3E). Kyoto Encyclopedia of Genes and Genomes (KEGG) pathway analysis (https://www.genome.jp/kegg/pathway.html) of the genes ranked by PCA loading showed a significant enrichment of several pathways, including insulin secretion and $\mathrm{Ca}^{2+}$ signaling (Table 1). These data illustrate that VSG rapidly regulates islet gene expression in a manner that is distinct from caloric restriction or sham-surgery. The pathways highlighted by this unbiased analysis suggest that bariatric surgery may enhance insulin secretion by altering intrinsic islet function, including $\mathrm{Ca}^{2+}$ signaling.

VSG enhanced intrinsic islet glucose sensitivity ex vivo. The remarkable differences in the islet transcriptome between VSG and AL- and PF-Sham controls led us to test the hypothesis that surgery induces intrinsic changes within the islet. To directly assess insulin secretion independently from differences in 
A

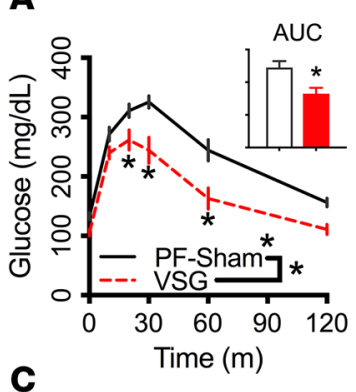

C

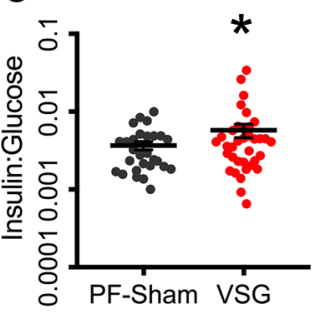

B

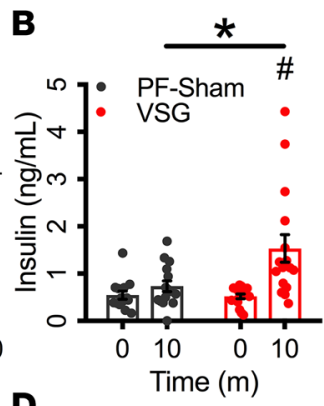

D

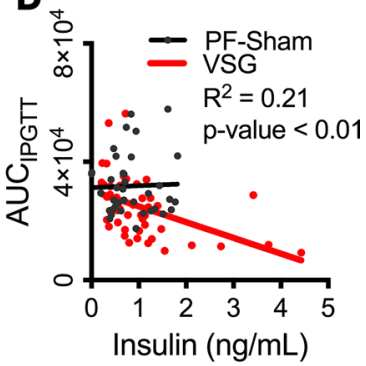

Figure 2. Glucose-stimulated insulin secretion is elevated after VSG. (A-C) Blood glucose and integrated AUC (A), circulating insulin (B), and insulin/glucose ratio (C) during an i.p. glucose tolerance test (PF-Sham, $n=14-29 ;$ VSG, $n=$ 16-36). (D) Correlation between insulin secretion ( $\mathrm{ng} / \mathrm{ml}$ ) and glucose excursion (AUC $\mathrm{IPGT}$; PF-Sham, $n=50 ; \mathrm{VSG}, n=59$ ) during an i.p. glucose tolerance test. PF-Sham animals are shown with black solid lines or gray circles; VSG animals are shown in red dashed lines or red circles. Data represent mean $\pm \mathrm{SEM}$; ${ }^{*} P<0.05$ between PF-Sham and VSC groups; ${ }^{\#} P$ $<0.05$ between time points within groups.

the systemic milieu that exist between groups, we isolated islets from PF-Sham and VSG animals and measured insulin secretion by perifusion. Islets from VSG mice displayed higher insulin secretion than those isolated from PF-Sham mice when exposed to stepwise elevations in glucose (Figure 4A). The insulin secretion rate is increased in response to $12 \mathrm{mM}$ glucose (Figure $4 \mathrm{~B}$ ) and shows a reduced $\mathrm{EC}_{50}$ $(\sim 30 \%, P \leq 0.001$; Figure 4C) for VSG compared with PF-Sham controls and a trend toward increased peak insulin secretion (Figure 4D). This effect is consistent in larger samples sizes (PF-Sham, 29; VSG, 39), where insulin secretion in response to the change from $2.7-10 \mathrm{mM}$ glucose was elevated in VSG islets compared with PF-Sham controls ( $~ 53 \%$ increase by integrated insulin AUC, $p=0.013$; Figure 4, $\mathrm{E}$ and $\mathrm{F}$ ). Insulin secretion and insulin AUC in response to depolarization by $30 \mathrm{mM} \mathrm{KCl}$ did not differ between groups, suggesting that insulin secretory capacity did not differ (Figure $4, \mathrm{G}$ and $\mathrm{H}$ ). Mechanisms linking glucose sensing to insulin secretion are affected (Figure 4, I-M). Representative $\mathrm{Ca}^{2+}$ traces (Figure 4I), plateau fraction (Figure 4J), and period (Figure 4K) demonstrate significant enhancements in the pulse duration and frequency $(24,25)$ of glucose-stimulated $\mathrm{Ca}^{2+}$ oscillations in VSG islets in 3 independent experiments, with a trend toward increased $\mathrm{Ca}^{2+}$ oscillation amplitude (Figure $4 \mathrm{~L}$ ). The average plateau fraction and period for each animal were also significantly different between groups; the oscillatory amplitude was unchanged (Supplemental Figure 2, A-C). In addition, the fraction of islets recruited by $7 \mathrm{mM}$ glucose increased by $>2$-fold in VSG animals relative to PF-Sham controls (Figure 4M), consistent with the left-shifted insulin secretory response (Figure 4C). These adaptations in VSG mice were not due to increased insulin content per islet or islet composition (i.e. percentage of area composed of $\beta$ cells or $\alpha$ cells) - none of which were different between groups (Figure 4, N-P).

Islet morphology and energetics were unchanged in the early postoperative period. $\beta$ Cell area and $\alpha$ cell area were comparable in surgery and control groups (Supplemental Figure 2, E and F). Representative images of islets from a PF-Sham or VSG mice are shown for reference (Supplemental Figure 2D). Oxygen consumption rate (OCR), determined in whole islets (Supplemental Figure 2G) and permeabilized islets (Supplemental Figure 2H) was not different between PF-Sham or VSG mice. Similarly, ATP synthesis and the ATP synthesis/OCR ratio were unchanged between groups in permeabilized islets (Supplemental Figure 2, I and J). 

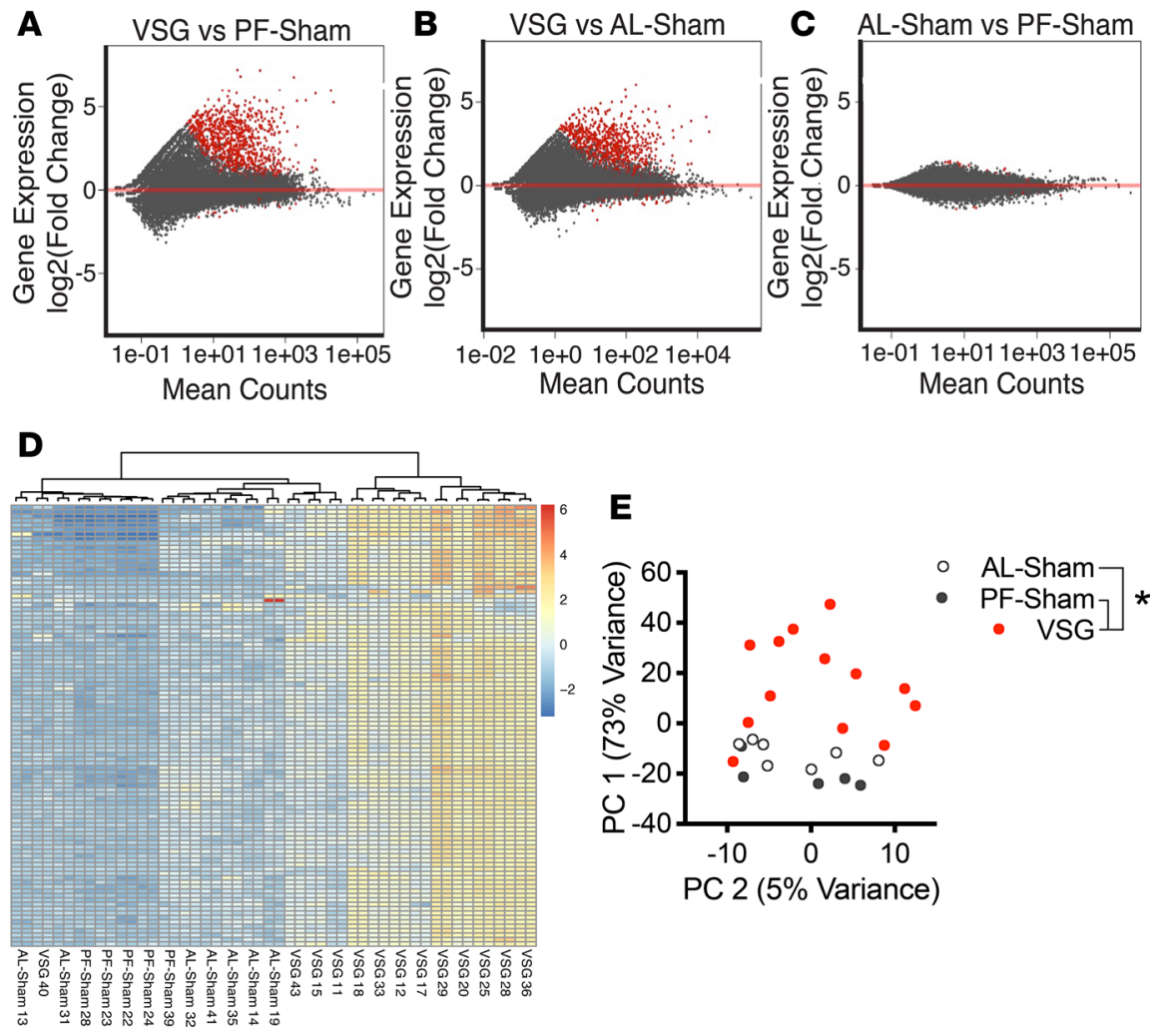

Figure 3. VSC significantly and uniquely modulates the islet transcriptome. (A-C) Volcano plots, where red points denoted significantly differentially expressed genes (DEG; Bonferroni-adjusted $P<0.05$ ) comparing islets from VSG ( $n$ $=13)$ and PF-Sham $(n=5)(\mathbf{A})$, VSG and AL-Sham $(n=7)(\mathbf{B})$, and PF-Sham and AL-Sham (C) groups. (D) Heatmap of the $\log _{2}$ transformed gene expression values for the top 100 most variant genes between groups and hierarchical clustering analysis of the most closely related samples. (E) Scatter plot showing a principal components analysis of the transcriptional data for AL-Sham (white circles), PF-Sham (gray circles), and VSG (red circles) islets. ${ }^{*} P<0.05$ between AL-Sham or PF-Sham and VSG groups when compared by 1-way ANOVA.

\section{Discussion}

It is well established that bariatric surgeries such as VSG improve glucose control. This is clearly seen in diabetic patients who experience reductions in both fasting and prandial glucose levels after surgery $(1,26)$. Surgical improvements to glucose control are largely attributed to reduced BW and improved insulin resistance $(27,28)$. Although the glucose-lowering effects of RYGB have been attributed entirely to weight-loss in some studies (11-13), it has long been observed that surgery lowers blood glucose in diabetic patients prior to significant weight loss $(15,16)$. This suggests that, while weight loss after surgery certainly enhances insulin sensitivity and improves glycemia (29-31), the early postsurgical glucoselowering effects of bariatric procedures may not be fully accounted for by caloric restriction in all cases (32). However, the mechanisms underlying these rapid, weight-independent outcomes of bariatric surgery are not fully understood. To address this question, we used a mouse model of bariatric surgery that allowed a comparison with a control group that could be matched carefully for caloric intake and BW; these PF-Sham mice control for the effects of VSG on energy balance that is difficult to achieve in human studies. Our findings paralleled clinical observations, as VSG mice displayed improved glucose tolerance and insulin secretion in vivo within 2 weeks of surgery, independent of differences in body weight or insulin sensitivity between groups. Islets from VSG mice displayed a unique gene expression profile that was enriched for pathways associated with enhanced insulin secretion, and they displayed ex vivo islet function that was augmented relative to controls. Collectively, these data indicate that there is a rapid adaptation of islet function after VSG that promotes glucose clearance independently of energy balance.

The weight-independent effects of bariatric surgery on blood glucose, particularly among diabetic patients, has been observed by surgeons for decades and are documented in clinical studies $(10,20,33)$. However, there has been some debate as to whether this is just the result of an abrupt reduction in caloric 
Table 1. KEGG pathway enrichment of the genes ranked according to PC 1 loading

\begin{tabular}{lccc}
\hline KEGG ID & Name & P value & Genes \\
mmu04971 & Gastric acid secretion & 0.008 & Mylk, Adcy9, Camk2a, Car2 \\
mmu04621 & NOD-like receptor signaling pathway & 0.01 & Adcy9, Rab11b, Dctn4 \\
mmu04962 & Vasopressin-regulated water reabsorption & 0.011 & Adcy9, Cacna1c, Camk2a, Pclo \\
mmu04911 & Insulin secretion & 0.013 & Mylk, Adcy9, Cacna1c, Camk2a, Egfr, P2rx7 \\
mmu04020 & Calcium signaling pathway & 0.014 & Car2, Car13 \\
mmu00910 & Nitrogen metabolism & 0.014 & Adcy9, Cacna1c, Camk2a, Egfr \\
mmu04912 & GnRH signaling pathway & 0.015 & Dnm3, Adcy9, Ap2b1 \\
mmu04961 & Endocrine and other factor-regulated & 0.02 & Cdk6, Egfr, Rassf1 \\
mmu05223 & calcium reabsorption & & Mylk, Adcy9, Cacna1c, Camk2a, Egfr
\end{tabular}

intake (34-37). To study this effectively, we pair-fed a sham-operated control group to match the caloric intake of VSG operated mice (Supplemental Figure 4). This resulted in in similar BWs and responses to i.p. insulin injections (i.e., insulin sensitivity) between the groups. To estimate the time course over which surgery improves glucose tolerance, we conducted pilot studies comparing the VSG and PF-Sham groups at different time intervals after surgery and observed that, by 10-14 days, there were reliable improvements in glycemia, approximating the rapid clinical effects that have been so compelling in surgical patients. Comparison of the glycemic excursions and AUC between the preoperative studies and the PF-Sham mice demonstrate a clear effect of weight loss on glucose tolerance. However, the effects of VSG on glycemia and islet function surpass those of weight loss alone (Supplemental Figure 3) and are present in response to fixed and ad libitum enteral meals, as well as nonenteral hyperglycemia. Thus, VSG in mice recapitulates many of the key features of the rapid response to bariatric surgery in a model that can be readily interrogated at the tissue level.

The early postoperative effects of bariatric surgery on glucose control are generally associated with increases in gastric-emptying rate, nutrient absorption, and enteroendocrine cell stimulation (4, 8). Accordingly, VSG-operated mice in our studies exhibited distinct glucose excursions and prandial glucoregulatory hormone profiles similar to previously reported rodent $(15,38)$ and human studies $(10,33)$. During a MMTT, circulating glucose and insulin rapidly peaked, within 10 minutes, in VSG animals, followed by a dramatic reduction of blood glucose to baseline levels by 30 minutes. The effect of surgery to promote insulin secretion was most clear in the fasting-refeeding paradigm, in which the 2 groups had comparable blood glucose concentrations but VSG mice had nearly double the insulin concentrations. Prandial circulating total GLP-1, GIP, and glucagon concentrations were higher in the VSG mice during both experimental paradigms, in keeping with previous reports $(10,39)$. Thus, the salutary effects of VSG on glucose homeostasis after oral nutrient delivery seem to be due in great part to enhanced insulin secretion. Some of the heightened insulin output during enteral feeding after VSG is likely due to increased gastric emptying, which contributes to modified nutrient flux and enhanced incretin levels. However, the GLP-1 receptor is not necessary for the glucose-lowering effect of surgery (40-42), and the GIP receptor has not been studied. Furthermore, in most studies with single gene KO models, the effect of surgery on glucose tolerance is retained (40, 42-46), with several exceptions (47-49). This suggests the mechanisms involved in enhanced insulin secretion after bariatric surgery are complex and likely multifaceted.

Because insulin responses to i.v. glucose improve in patients with diabetes after bariatric surgery (19$21)$, we hypothesized that there is an intrinsic improvement in $\beta$ cell function postoperatively. To test this hypothesis, we measured glucose excursion and insulin responses during IPGTT, both of which do not engage the surgically modified gastrointestinal tract. By postoperative day 10, VSG mice had improved i.p. glucose tolerance compared with PF-Sham and AL-Sham (Supplemental Figure 3), with associated increases in GSIS (Figure 2B) and insulin/glucose ratio (Figure 2C). While glucose control is improved in both the PF-Sham and VSG group compared with the AL-Sham animals, there is a superior effect of VSG on glucose clearance, indicating that weight loss is not solely responsible for the improvements in glycemia. A large fraction of glucose disposal in mice is independent of insulin action, although insulin- 

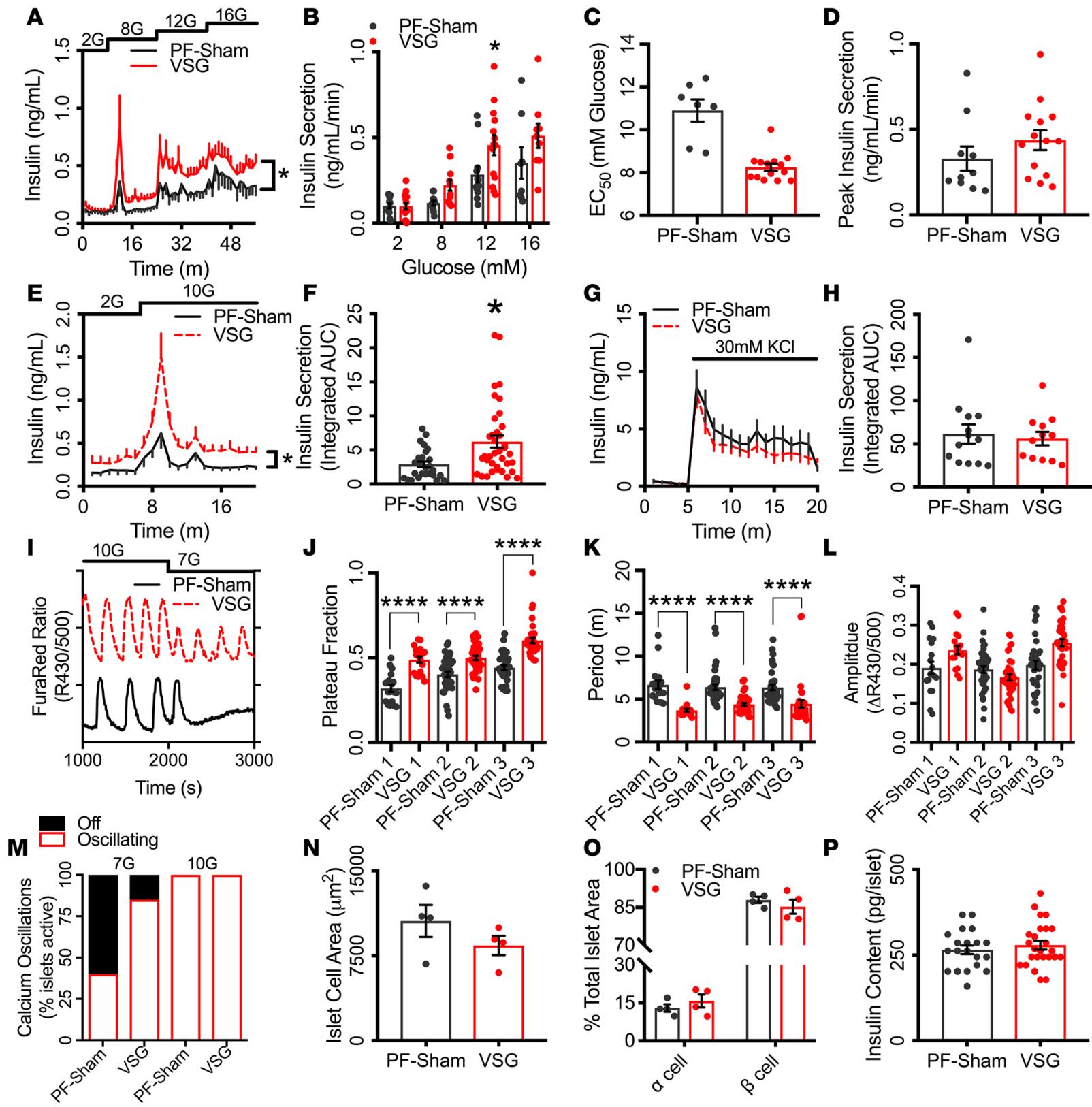

$\mathbf{P}$

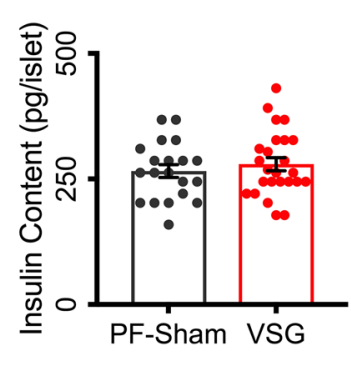

Figure 4. VSC enhanced intrinsic islet glucose sensitivity ex vivo. (A-D) Insulin secretion in response to step-wise elevations in glucose (A and $\mathbf{B})$, $E C_{50}$ (C), and peak insulin secretion (D) for an ex vivo perifusion glucose ramp exposing PF-Sham islets $(n=7)$ and VSG islets $(n=14)$ to $2,8,12$, and 16 mM glucose. (E and F) GSIS (E) and insulin AUC (F) from ex vivo perifusion experiments treating PF-Sham $(n=29)$ or VSG $(n=39)$ islets with $10 \mathrm{mM}$ glucose. (G and $\mathbf{H})$ Insulin secretion and insulin AUC from ex vivo perifusion experiments treating PF-Sham $(n=13)$ or VSG $(n=11)$ islets with $2 \mathrm{mM} \mathrm{glucose}+30 \mathrm{mM} \mathrm{KCl}$. (I-L) Representative $\mathrm{Ca}^{2+}$ traces (I) and plateau fraction (AUC of 50\% maximum amplitude) (J), period (K), and amplitude of Ca $\mathrm{a}^{2+}$ oscillations (L) in islets from PF-Sham ( $n=3$ mice) or VSC ( $n=3$ mice). (M) Percentage of islets undergoing active Ca ${ }^{2+}$ oscillations when treated with either $7 \mathrm{mM}$ glucose (7C) or $10 \mathrm{mM}$ glucose (10G). ( $\mathbf{N}$ and $\mathbf{0}$ ) Total islet area (N) and percentage of the islet composed of either $\beta$ cells or $\alpha$ cells (0) for PF-Sham and VSC mice ( $n=4$ per group). (P) Insulin content per islet from PF-Sham $(n=20)$ or VSG $(n=25)$. PF-Sham animals are shown with a solid gray line or gray circles; VSG animals are shown with a dashed red line or red circles. Data represent mean $\pm S E M ;{ }^{*} P<0.05$ and ${ }^{* * * *} P<0.0001$ between PF-Sham and VSC groups.

dependent processes are increased in states of hyperinsulinemia (50). This phenomenon was evident in the PF-Sham animals, where insulin secretion and glucose control were not significantly correlated. However, VSG significantly modified the normal physiology, as enhanced in vivo insulin secretion in response to i.p. glucose correlated with the IPGTT glucose AUC. These results demonstrate that, in mice, GSIS increases rapidly after surgery and is not wholly dependent on enteral nutrient flux or acute enteroendocrine regulation. An important implication of this finding is that $\beta$ cells adapt early to bariatric surgery through increased sensitivity to stimulation by ambient glucose concentrations. While these data 
are suggestive of a surgical enhancement to intrinsic islet glucose sensitivity, that interpretation is confounded by the presence of other insulinotropic factors in vivo, like glucagon, which appear to be elevated even in the fasting state after surgery.

The surgical effect to enhance in vivo GSIS independently of BW or acute incretin regulation raises the possibility that this trait is imprinted upon the islet by persistent changes in intrinsic function, which may be viewed through analysis of gene expression programs. In subsequent RNA-seq experiments, VSG islets exhibit 459 and 611 DEGs (FDR adjusted $P<0.05$ ) compared with AL-Sham and PF-Sham and groups, respectively. By contrast, there is a weaker effect of 10 days of food restriction on islet gene transcription, with only 38 genes achieving statistical significance between PF-Sham and AL-Sham groups. Furthermore, the limited effect of caloric restriction on islet gene transcription compared with those elicited by bariatric surgery (Figure 3A) demonstrates that postsurgical modification of the islet transcriptome is not simply a side effect of negative energy balance, but rather a unique facet of the metabolic adaptation to surgery. The hierarchical clustering and PCA demonstrated separation of the VSG group away from both sham-operated groups, while the PF-Sham and AL-Sham groups are much more similar. Thus, VSG conferred the islet with a unique transcriptional profile, which was presumably largely driven by modifications to $\beta$ cells transcripts, as these islets were composed of $\sim 85 \% \beta$ cells. Gene set enrichment analysis (GSEA) of the top 100 most variably expressed genes ranked by PCA loading values are enriched in KEGG pathways for not only insulin secretion, but also $\mathrm{Ca}^{2+}$ signaling - a requisite factor for insulin secretion (51). The canonical glucose-sensing machinery, including glucokinase and glucose transporters, are not significantly changed. While it is unclear what factor(s) unique to VSG drive these changes in islet gene expression, the dominant influence of genes such as Camk2a, Cacna1c, and Adcy9 in the KEGG pathway analysis suggests that ion balance and/or cAMP signaling may play a roll. Transcripts for GLP-1 receptor (Glp1r), GIP receptor (Gipr), and glucagon receptor (Gcgr) — G-protein coupled receptors known to stimulate cAMP - are not modified in the islet (Supplemental Table 1), indicating that sensitivity to acute incretin stimuli may not be enhanced at the receptor expression level after VSG. However, the role of $\alpha$ - to $\beta$ cell communication (i.e., $\alpha$ cell proglucagon products potentiating insulin release) remains unknown $(52,53)$. Ultimately, these data demonstrate a unique remodeling of the islet transcriptomic profile by VSG, which may functionally potentiate $\mathrm{Ca}^{2+}$ signaling and, subsequently, GSIS.

We next sought to test whether VSG islets demonstrate a functional improvement in insulin secretion when isolated from the in vivo environment. Islets isolated from VSG mice displayed significantly elevated GSIS and a left-shifted insulin response to glucose compared with controls, consistent with increased $\beta$ cell sensitivity to glucose. This secretory profile was corroborated by increased intra- $\beta$-cellular $\mathrm{Ca}^{2+}$ fluxes in response to increased glucose exposure, a compelling finding insofar as $\mathrm{Ca}^{2+}$ is a critical mediator of insulin exocytosis $(24,51)$. We focused on the calcium plateau fraction, the fraction of time spent in the "on" state of an oscillation, and the mechanism by which glucose sensing is encoded in the $\beta$ cell metabolic and calcium oscillations $(24,25)$. Based on the glucose dependence of the calcium plateau fraction (54), it can be estimated that VSG has the equivalent effect of increasing extracellular glucose by $\sim 3 \mathrm{mM}$. Consequently, the percentage of islets exhibiting calcium oscillations in response to $7 \mathrm{mM}$ glucose was also enhanced 2.2 fold in the VSG group, further indicating increased islet glucose sensitivity after surgery. These differences are large enough to account for the left-shift in glucose threshold observed in the ex vivo GSIS perifusion studies and indicate an intrinsic improvement of $\beta$ cell function following VSG.

These results from isolated islets provide a direct link to the islet transcriptional profile, with its enriched $\mathrm{Ca}^{2+}$ signaling pathway and elevated insulin secretory function. Given that 1 mechanism connecting islet glucose sensitivity to insulin release is via the canonical metabolic amplifying pathways $(24,55)$, we investigated various elements of this pathway. Overall, mitochondrial function as determined by 2 separate measurements of islet $\mathrm{OCR}\left(\mathrm{JO}_{2}\right)$, ATP synthesis, and ATP synthesis/OCR were unchanged by surgery. Thus, enhanced islet mitochondrial oxidative capacity does not seem to explain the enhanced $\beta$ cell function. Islet architecture (e.g., islet size, islet area composed of $\alpha$ - or $\beta$ cells, and $\alpha$ - and $\beta$ cell mass) also did not differ between groups, suggesting that islet mass and morphology do not contribute to the differences in islet function shown here. This is reinforced by the finding that neither $\alpha$ cell- or $\beta$ cell-specific gene transcripts are notably enriched in our transcriptomic analysis (Supplemental Table 3), but rather that there is a largely uniform enrichment of genes that are expressed in both $\alpha$ - and $\beta$ cells (56).

To our knowledge, only 1 previous study has assessed ex vivo islet insulin secretion after VSG in mice, in which the investigators found no differences between the $d b / d b$ VSG and $d b / d b$ PF-Sham controls (57). There are a number of possible explanations for the difference between our data and those presented by Abu-Gazala 
et al. First, they studied a mouse strain with absent leptin signaling and dysfunctional islets marked by lipid infiltration and disrupted architecture $(58,59)$. Although the leptin receptor is not robustly expressed in mouse islets and seems unlikely to be the direct cause of $\alpha$ - or $\beta$ cell impairment (60), indirect effects mediated through the systemic impact of leptin signaling deficiency could confound beneficial effects mediated through the islet. Testing this possibility would require comparison of islet from $d b / d b$ and diet-induced obesity mice before surgery. Second, we studied animals 10-14 days after surgery and Abu-Gazala and colleagues (57) did their studies at 30 days. It is possible that the effects of VSG change and adapt over time. This is well described in humans with VSG, where enhancement of insulin secretion occurs early after surgery but wanes as insulin sensitivity increases over time (10). While our results and those of Abu-Gazala appear, at first glance, to be diametrically opposed, differences in animal background and length of time after surgery provide plausible explanations as to how they might be compatible. Certainly, these provide useful hypotheses for future study.

Our findings collectively illustrate that VSG fundamentally sensitizes the islet to glucose. This may serve as a critical adaptation to cope with the homeostatic challenge presented by the increased flux of nutrients from the gut to the circulation common to many bariatric surgeries (4). The sensitizing effect is intrinsic to islets adapted to surgery for 10 days, as it persists ex vivo apart from any VSG-mediated, islet-extrinsic factors. This effect is concurrent with an amplified $\mathrm{Ca}^{2+}$ response to glucose that appears to be imprinted upon the islet transcriptomic profile. Critically, the enhancement of islet function in the early postoperative period is a significant driver of the early, weight-independent improvements to glucose control that have garnered intense interest throughout the diabetes field. This discovery suggests factors unleashed early in the course of VSG, and possibly other bariatric surgeries, have direct effects on $\beta$ cell function. Understanding and harnessing these factors has potential for recovering insulin secretion in diabetic patients.

\section{Methods}

Animals. WT mice (C57BL/6J; The Jackson Laboratory) were bred in-house at the Duke Molecular Physiology Institute and placed on HFD (Research Diets Inc., 45\% kcal from lipid, catalog D12451) at 8 weeks of at an average BW of $\sim 19 \mathrm{~g}$. Animals were weighed once weekly for 56 days ( 9 weeks) until they achieved BW of $\sim 38 \mathrm{~g}$. Animals were divided into separate groups with equivalent metabolic profiles based on BW (Supplemental Figure 1A), preoperative IPGTT (Supplemental Figure 1B), and preoperative MMTT (Supplemental Figure 1D). Glucose curves and circulating insulin concentrations (Supplemental Figure 1, B-E) were not different between groups. A total of 70 mice were subjected to VSG (60 survivors; 10 died, were sacrificed, or were discarded), and 60 mice received Sham surgery and pair-feeding (53 survivors; 7 died, were sacrificed, or were discarded). Mice were sacrificed or discarded due to illness, excess BW loss (> 20\% BW), or hypoglycemia (fasting blood glucose < 35mg/dl).

Surgeries. Two days prior to surgery, mice were moved from cages containing corn-cob bedding to ones containing autoclaved paper bedding with enrichment and given $\sim 20 \mathrm{ml}$ liquid Ensure Plus (Abbot) as their daily food ration. The day before surgery, animals were placed in fresh cages and fasted overnight. Before surgery, they received BuprenexSQ (50 $\mu$, compounded by ZooPharm), Meloxicam ( $0.5 \mathrm{mg} / \mathrm{kg}$, Apotex), and Gentamicin ( $\sim 8 \mathrm{mg} / \mathrm{kg}$, VetOne) as i.p. injections. Procedures were performed under isoflurane anesthesia, starting with a midline skin incision $(1.5 \mathrm{~cm}$ in length) below the xyphoid process, which was then repeated in the underlying muscle of the body wall. The stomach and spleen were delivered through the incision using a pair of sterile cotton swaps, and the spleen was separated from the greater curvature of the stomach, taking care not to compromise major blood vessels. The suspensory ligament was incised at its attachment to the cardia, increasing the angle of His. A small incision was made in the membranous fundus along the greater curvature, and stomach contents were extruded by gentle pressure with sterile cotton swaps, ensuring not to contaminate the surgical field. A stainless steel Ligaclip (LS-400, Ethicon) was introduced at the angle of His, clamped across the stomach, and the $70 \%-80 \%$ of remnant stomach outside the tube formed by the clip was excised. Three sutures (6-0 Ethilon, Ethicon) were placed around the clip and through both walls of the stomach ( 1 at each end and 1 in the center). The body wall was closed with a continuous 4-0 Vicryl suture (Ethicon), and skin was closed in a continuous, s.c. fashion using identical suture material. Sham-operated control mice had laparotomy, with stomach isolation and temporary removal, before securing the gastrointestinal tract back in the abdomen and closing the incision. VSG and sham operations were performed in parallel, with some mice receiving one or the other procedure on the same days of surgery. 
Postoperative experimental paradigm. All animals received daily meloxicam and saline injections for 2 days after surgery and were kept on liquid diet for 4 days after surgery. On day 5, animals were given solid, standard chow for 1 day, and they were then switched back to HFD on day 6 . Individual BW was measured daily for the first 14 days after surgery, while food consumption was measured daily from postoperative days 5-14. Animals receiving sham operations received a daily food ration equivalent to that consumed by the VSG group on the previous day between 16:00-17:00 hours. The PF-Sham animals generally consumed the entire daily ration within the first hour (i.e., 17:00-18:00 hours). In vivo assessments were performed as follows: IPGTT (post-operative day 10), MMTT or insulin tolerance test (post-operative day 12), and overnight fast/30-minute refeed (post-operative day 14). These experiments were staggered across cohorts so that no animal was fasted and tested twice within 3 days. Methods for these tests have been reported previously $(61,62)$. IPGTTs were performed in mice fasted for 5 hours (0800-1300 hours) using a $1.5 \mathrm{~g} /$ $\mathrm{kg}$ glucose dissolved in PBS (Thermo Fisher Scientific, catalog 10010023). Blood glucose was measured at $\mathrm{t}=0,10,20,30,60,90$, and 120 minutes and blood ( $25 \mu 1$ for IPGTTs, $\sim 100 \mu 1$ for MMTT, and overnight fast/30-minute refeed) was collected at 0 and 10 minutes in EDTA-coated tubes (Microvette, catalog NC9299309). ITTs were performed in mice fasted for 5 hours (0800-1300 hours) using an insulin dose of $0.5 \mathrm{U} / \mathrm{kg}$ (Humalog, Lilly). Blood glucose was measured at $\mathrm{t}=0,10,20,30,60,90,120$, and 150 minutes, and blood ( $\sim 25 \mu \mathrm{l})$ was collected at 0 and 45 minutes. MMTTs were performed in mice fasted for 5 hours (0800-1300 hours) using an oral gavage of $200 \mu 1$ Ensure Plus, measuring blood glucose at $\mathrm{t}=0,10,20$, $30,60,90$, and 120 minutes, and collecting blood glucose at $t=0$ and 10 minutes. Overnight fast, refeeding studies were performed in mice fasted for 16 hours (1700-0900 hours). Fasting blood glucose and a fasting blood sample were taken before allowing the mouse to consume a pellet of HFD ad libitum for 30 minutes. A refed blood glucose and refed blood sample were taken, food consumption was measured, and the animal was sacrificed for islet harvest.

Peptide assays. Tail-vein blood glucose for IPGTT and MMTT was assayed in real time using Bayer Contour Glucometer. Plasma was separated by centrifugation (1000 g, 15 minutes) at $4^{\circ} \mathrm{C}$ and stored at $-20^{\circ} \mathrm{C}$. The following serum peptides were assayed according to protocols suggested by the manufacturer as follows: insulin (CrystalChem, catalog 90080), total GLP-1 (Meso Scale Discovery, catalog K150JVC-1), GIP (CrystalChem, catalog 81517), and glucagon (Mercodia, catalog 10-1271-01). Perifusion samples were assayed for insulin according to protocols provided by the manufacturer as follows: insulin (Perkin-Elmer, catalog AL-204).

Islet isolation. Isolation of primary mouse islets is described elsewhere (61). Briefly, the pancreas was inflated via the pancreatic duct with type V collagenase $(0.8 \mathrm{mg} / \mathrm{ml}$, MilliporeSigma, catalog C5138), excised, and digested for 12 minutes at $37^{\circ} \mathrm{C}$. The digest was washed with cold RPMI (10 mM glucose [Sigma], $2 \mathrm{mM}$ L-glutamine [Sigma], $100 \mathrm{U} / \mathrm{ml}$ penicillin [Thermo Fisher], 0.25\% BSA [Sigma], and 100 $\mu \mathrm{g} / \mathrm{ml}$ streptomycin [Thermo Fisher]), before separating islets using a standard Histopaque gradient. Islets were either allowed to recover overnight in RPMI with $10 \% \mathrm{FBS}$ at $37^{\circ} \mathrm{C}$ for further ex vivo perifusion experiments or placed in TRI Reagent mRNA isolation.

$R N A$ isolation, sequencing, and analysis. Islet mRNA was isolated by standard trireagent protocols as described previously (63). Illumina stranded mRNA-seq libraries and HiSeq $400050 \mathrm{bp}$ sequencing was performed in duplicate for each sample by the Duke Center for Genomic and Computational Biology. Quality control of .fasta files (FastQC), trimming adapters (TrimGalore), alignment to the genome (Bowtie2), and nonnormalized gene counts (featureCounts) were all performed on the Galaxy web platform using the public server at usegalaxy.org to analyze the data (64). Downstream differential gene expression and PCA were performed using DESeq2 (65) and pcaExplorer (66), respectively. GSEA was performed using WebGestalt (67).

Islet perifusion. Islet perifusion experiments were performed using the Biorep Perifusion apparatus with 50 islets per chamber that had been cultured overnight in RPMI (Life Technologies) with 10\% FBS (Sigma) at $37^{\circ} \mathrm{C}$. All perifusion experiments were carried out in a base $\mathrm{KRPH}$ buffer $(135 \mathrm{mM} \mathrm{NaCl}, 3.6 \mathrm{mM} \mathrm{KCl}$, $1.5 \mathrm{mM} \mathrm{CaCl}_{2}, 0.5 \mathrm{mM} \mathrm{NaH}_{2} \mathrm{PO}_{4}, 0.5 \mathrm{mM} \mathrm{MgSO}_{4}, 5 \mathrm{mM}$ HEPES, $5 \mathrm{mM} \mathrm{NaCO}, 0.1 \%$ BSA, pH 7.5, Sigma), with $200 \mu \mathrm{l} / \mathrm{m}$ flow rate, and preceded by a 48 -minute equilibration period with $\mathrm{KRPH}+2 \mathrm{mM}$ glucose. All samples were stored at $-20^{\circ} \mathrm{C}$ before being assayed for insulin.

Glucose ramp. Islets from PF-sham $(n=12)$ and VSG $(n=15)$ were treated with $2 \mathrm{mM}$ glucose for 8 minutes, followed by 16 -minute treatments with 8,12 , and $16 \mathrm{mM}$ glucose. Insulin secretion rates was calculated as the $\mathrm{ng} / \mathrm{ml}$ insulin secreted per minute of treatment. Potassium Chloride treatment was performed in both PF-sham and VSG islets under $2 \mathrm{mM}$ glucose conditions using $30 \mathrm{mM} \mathrm{KCl}$. 
Mitochondrial function. Oxygen consumption was assessed in intact islets using Agilent Seahorse according to previously published protocols (68), while mitochondrial function and ATP synthesis were assessed in permeabilized islets using the Oroboros Oxygraph-2K (Oroboros Instruments) and fluorometry in accordance with previously published protocols $(69,70)$

$\mathrm{Ca}^{2+}$ flux. Calcium oscillation traces and calculations were performed in whole islets exposed to $10 \mathrm{mM}$ glucose in accordance with previously published protocols (54).

Statistics. All data are presented as mean \pm SEM and were analyzed using Graphpad Prism unless otherwise noted in the figure legends. Integrated AUC was calculated using the fasting blood glucose of each animal as their own baseline and compared between groups using a 2-tailed, unpaired $t$ test. A linear regression was used to analyze the relationship between glucose AUC and in vivo GSIS in Figure 2D; the slope of the line is compared with a hypothetical slope of 0 . All other data were analyzed using 1-way ANOVA or 2-way ANOVA where appropriate. A post-hoc Sidak's multiple comparison test was used to correct for multiple comparisons.

Study approval. All procedures involving animals were approved by Duke IACUC and Duke Laboratory Animal Resource Division.

\section{Author contributions}

JDD, KFW, EP, MJM, JC, AM, and DD designed research studies; JDD, JN, SS, TG, KFW, MB, RA, AM, and MM conducted experiments; JDD, KFW, EP, MJM, MAH, JT, JC, and DD analyzed data; JDD and DD wrote the manuscript.

\section{Acknowledgments}

JDD received funding from T32DK007012, 1F32DK115031-01, Duke-NUS Collaborative Research Initiative Trainee Exchange Program. MJM received funding from American Diabetes Association (1-16IBS-212), the NIH/NIDDK (R01DK113103), the NIH/NIA (R21AG050135, R01AG062328), and a New Investigator Award from the Wisconsin Partnership Program.

Address correspondence to: Jonathan D. Douros, 300 N. Duke Street, Carmichael Building, 49-108I, Durham North Carolina, 27701, USA. Phone: 919.684.5418; Email: jonathan.douros@duke.edu.

BMB's present address is: Agilent Technologies, Lexington, Massachusetts, USA.

1. Schauer PR, et al. Bariatric surgery versus intensive medical therapy for diabetes--3-year outcomes. $N$ Engl J Med. 2014;370(21):2002-2013.

2. Buchwald H, et al. Bariatric surgery: a systematic review and meta-analysis. JAMA. 2004;292(14):1724-1737.

3. Braghetto I, et al. Scintigraphic evaluation of gastric emptying in obese patients submitted to sleeve gastrectomy compared to normal subjects. Obes Surg. 2009;19(11):1515-1521.

4. Chambers AP, et al. Regulation of gastric emptying rate and its role in nutrient-induced GLP-1 secretion in rats after vertical sleeve gastrectomy. Am J Physiol Endocrinol Metab. 2014;306(4):E424-E432.

5. Bernstine $\mathrm{H}$, et al. Gastric emptying is not affected by sleeve gastrectomy--scintigraphic evaluation of gastric emptying after sleeve gastrectomy without removal of the gastric antrum. Obes Surg. 2009;19(3):293-298.

6. Bojsen-Møller KN, et al. Accelerated protein digestion and amino acid absorption after Roux-en-Y gastric bypass. Am J Clin Nutr. 2015;102(3):600-607.

7. Cavin JB, et al. Differences in Alimentary Glucose Absorption and Intestinal Disposal of Blood Glucose After Roux-en-Y Gastric Bypass vs Sleeve Gastrectomy. Gastroenterology. 2016;150(2):454-64.e9.

8. Dirksen C, et al. Fast pouch emptying, delayed small intestinal transit, and exaggerated gut hormone responses after Roux-en-Y gastric bypass. Neurogastroenterol Motil. 2013;25(4):346-e255.

9. Gehrer S, Kern B, Peters T, Christoffel-Courtin C, Peterli R. Fewer nutrient deficiencies after laparoscopic sleeve gastrectomy (LSG) than after laparoscopic Roux-Y-gastric bypass (LRYGB)-a prospective study. Obes Surg. 2010;20(4):447-453.

10. Nannipieri M, et al. Roux-en-Y gastric bypass and sleeve gastrectomy: mechanisms of diabetes remission and role of gut hormones. J Clin Endocrinol Metab. 2013;98(11):4391-4399.

11. Jackness $C$, et al. Very low-calorie diet mimics the early beneficial effect of Roux-en-Y gastric bypass on insulin sensitivity and $\beta$-cell Function in type 2 diabetic patients. Diabetes. 2013;62(9):3027-3032.

12. Lips MA, et al. Calorie restriction is a major determinant of the short-term metabolic effects of gastric bypass surgery in obese type 2 diabetic patients. Clin Endocrinol (Oxf). 2014;80(6):834-842.

13. Steven $\mathrm{S}$, et al. Calorie restriction and not glucagon-like peptide-1 explains the acute improvement in glucose control after gastric bypass in Type 2 diabetes. Diabet Med. 2016;33(12):1723-1731.

14. Jørgensen NB, et al. Acute and long-term effects of Roux-en-Y gastric bypass on glucose metabolism in subjects with Type 2 diabetes and normal glucose tolerance. Am J Physiol Endocrinol Metab. 2012;303(1):E122-E131.

15. Chambers AP, et al. Weight-independent changes in blood glucose homeostasis after gastric bypass or vertical sleeve gastrectomy 
in rats. Gastroenterology. 2011;141(3):950-958.

16. Laferrère B, et al. Effect of weight loss by gastric bypass surgery versus hypocaloric diet on glucose and incretin levels in patients with type 2 diabetes. J Clin Endocrinol Metab. 2008;93(7):2479-2485.

17. Laferrère B. Do we really know why diabetes remits after gastric bypass surgery? Endocrine. 2011;40(2):162-167.

18. Laferrère B. Diabetes remission after bariatric surgery: is it just the incretins? Int J Obes (Lond). 2011;35 Suppl 3:S22-S25.

19. Polyzogopoulou EV, Kalfarentzos F, Vagenakis AG, Alexandrides TK. Restoration of euglycemia and normal acute insulin response to glucose in obese subjects with type 2 diabetes following bariatric surgery. Diabetes. 2003;52(5):1098-1103.

20. Kashyap SR, et al. Acute effects of gastric bypass versus gastric restrictive surgery on beta-cell function and insulinotropic hormones in severely obese patients with type 2 diabetes. Int J Obes (Lond). 2010;34(3):462-471.

21. Lin E, et al. Improvement in ß-cell function in patients with normal and hyperglycemia following Roux-en-Y gastric bypass surgery. Am J Physiol Endocrinol Metab. 2010;299(5):E706-E712.

22. Kahn SE. The relative contributions of insulin resistance and beta-cell dysfunction to the pathophysiology of Type 2 diabetes. Diabetologia. 2003;46(1):3-19.

23. Nosso G, et al. Comparative Effects of Roux-en-Y Gastric Bypass and Sleeve Gastrectomy on Glucose Homeostasis and Incretin Hormones in Obese Type 2 Diabetic Patients: A One-Year Prospective Study. Horm Metab Res. 2016;48(5):312-317.

24. Henquin JC. Regulation of insulin secretion: a matter of phase control and amplitude modulation. Diabetologia. 2009;52(5):739-751.

25. Miura RM, Pernarowski M. Correlations of rates of insulin release from islets and plateau fractions for beta-cells. Bull Math Biol. 1995;57(2):229-246.

26. Schauer PR, et al. Bariatric Surgery versus Intensive Medical Therapy for Diabetes - 5-Year Outcomes. $N$ Engl J Med. 2017;376(7):641-651.

27. Gumbs AA, Modlin IM, Ballantyne GH. Changes in insulin resistance following bariatric surgery: role of caloric restriction and weight loss. Obes Surg. 2005;15(4):462-473.

28. Abu-Gazala S, et al. Sleeve Gastrectomy Improves Glycemia Independent of Weight Loss by Restoring Hepatic Insulin Sensitivity. Diabetes. 2018;67(6):1079-1085.

29. Bojsen-Møller KN, et al. Early enhancements of hepatic and later of peripheral insulin sensitivity combined with increased postprandial insulin secretion contribute to improved glycemic control after Roux-en-Y gastric bypass. Diabetes. 2014;63(5):1725-1737

30. Lima MM, et al. Acute effect of roux-en-y gastric bypass on whole-body insulin sensitivity: a study with the euglycemic-hyperinsulinemic clamp. J Clin Endocrinol Metab. 2010;95(8):3871-3875.

31. Severino A, et al. Early effect of Roux-en-Y gastric bypass on insulin sensitivity and signaling. Surg Obes Relat Dis. 2016;12(1):42-47.

32. Berggren J, Lindqvist A, Hedenbro J, Groop L, Wierup N. Roux-en-Y gastric bypass versus calorie restriction: support for surgery per se as the direct contributor to altered responses of insulin and incretins to a mixed meal. Surg Obes Relat Dis. 2017;13(2):234-242.

33. Dirksen C, et al. No Islet Cell Hyperfunction, but Altered Gut-Islet Regulation and Postprandial Hypoglycemia in Glucose-Tolerant Patients 3 Years After Gastric Bypass Surgery. Obes Surg. 2016;26(9):2263-2267.

34. Campos GM, et al. Improvement in peripheral glucose uptake after gastric bypass surgery is observed only after substantial weight loss has occurred and correlates with the magnitude of weight lost. J Gastrointest Surg. 2010;14(1):15-23.

35. Foo J, et al. Studies in insulin resistance following very low calorie diet and/or gastric bypass surgery. Obes Surg. 2011;21(12):1914-1920.

36. Gastaldelli A, et al. Short-term Effects of Laparoscopic Adjustable Gastric Banding Versus Roux-en-Y Gastric Bypass. Diabetes Care. 2016;39(11):1925-1931.

37. Pop LM, et al. Roux-en-Y gastric bypass compared with equivalent diet restriction: Mechanistic insights into diabetes remission. Diabetes Obes Metab. 2018;20(7):1710-1721.

38. Pressler JW, et al. Vertical sleeve gastrectomy restores glucose homeostasis in apolipoprotein A-IV KO mice. Diabetes. 2015;64(2):498-507.

39. Mallipedhi A, Prior SL, Barry JD, Caplin S, Baxter JN, Stephens JW. Temporal changes in glucose homeostasis and incretin hormone response at 1 and 6 months after laparoscopic sleeve gastrectomy. Surg Obes Relat Dis. 2014;10(5):860-869.

40. Douros JD, et al. Enhanced Glucose Control Following Vertical Sleeve Gastrectomy Does Not Require a $\beta$-Cell Glucagon-Like Peptide 1 Receptor. Diabetes. 2018;67(8):1504-1511.

41. Garibay D, et al. $\beta$-Cell Glucagon-Like Peptide-1 Receptor Contributes to Improved Glucose Tolerance After Vertical Sleeve Gastrectomy. Endocrinology. 2016;157(9):3405-3409.

42. Wilson-Pérez HE, et al. Vertical sleeve gastrectomy is effective in two genetic mouse models of glucagon-like Peptide 1 receptor deficiency. Diabetes. 2013;62(7):2380-2385.

43. Chambers AP, et al. The effects of vertical sleeve gastrectomy in rodents are ghrelin independent. Gastroenterology. 2013;144(1):50-52.e5.

44. Mul JD, et al. MGAT2 deficiency and vertical sleeve gastrectomy have independent metabolic effects in the mouse. Am JPhysiol Endocrinol Metab. 2014;307(11):E1065-E1072.

45. Arble DM, Pressler JW, Sorrell J, Wevrick R, Sandoval DA. Sleeve gastrectomy leads to weight loss in the Magel2 knockout mouse. Surg Obes Relat Dis. 2016;12(10):1795-1802.

46. Mokadem M, Zechner JF, Uchida A, Aguirre V. Leptin Is Required for Glucose Homeostasis after Roux-en-Y Gastric Bypass in Mice. PLoS One. 2015;10(10):e0139960.

47. Ding L, et al. Vertical sleeve gastrectomy activates GPBAR-1/TGR5 to sustain weight loss, improve fatty liver, and remit insulin resistance in mice. Hepatology. 2016;64(3):760-773.

48. Ryan KK, et al. FXR is a molecular target for the effects of vertical sleeve gastrectomy. Nature. 2014;509(7499):183-188

49. McGavigan AK, et al. TGR5 contributes to glucoregulatory improvements after vertical sleeve gastrectomy in mice. Gut. 2017;66(2):226-234.

50. Pacini G, Thomaseth K, Ahrén B. Contribution to glucose tolerance of insulin-independent vs. insulin-dependent mechanisms 
in mice. Am J Physiol Endocrinol Metab. 2001;281(4):E693-E703.

51. Grodsky GM, Bennett LL. Cation requirements for insulin secretion in the isolated perfused pancreas. Diabetes. 1966;15(12):910-913.

52. Garibay D, et al. $\beta$ Cell GLP-1R Signaling Alters $\alpha$ Cell Proglucagon Processing after Vertical Sleeve Gastrectomy in Mice. Cell Rep. 2018;23(4):967-973.

53. Svendsen B, et al. Insulin Secretion Depends on Intra-islet Glucagon Signaling. Cell Rep. 2018;25(5):1127-1134.e2.

54. Gregg T, et al. Pancreatic $\beta$-Cells From Mice Offset Age-Associated Mitochondrial Deficiency With Reduced KATP Channel Activity. Diabetes. 2016;65(9):2700-2710.

55. MacDonald PE, Joseph JW, Rorsman P. Glucose-sensing mechanisms in pancreatic beta-cells. Philos Trans R Soc Lond, B, Biol Sci. 2005;360(1464):2211-2225.

56. DiGruccio MR, et al. Comprehensive alpha, beta and delta cell transcriptomes reveal that ghrelin selectively activates delta cells and promotes somatostatin release from pancreatic islets. Mol Metab. 2016;5(7):449-458.

57. Abu-Gazala S, et al. Sleeve Gastrectomy Improves Glycemia Independent of Weight Loss by Restoring Hepatic Insulin Sensitivity. Diabetes. 2018;67(6):1079-1085.

58. Covey SD, et al. The pancreatic beta cell is a key site for mediating the effects of leptin on glucose homeostasis. Cell Metab. 2006;4(4):291-302.

59. Ravier MA, Sehlin J, Henquin JC. Disorganization of cytoplasmic $\mathrm{Ca}(2+)$ oscillations and pulsatile insulin secretion in islets from ob/ obmice. Diabetologia. 2002;45(8):1154-1163.

60. Soedling H, et al. Limited impact on glucose homeostasis of leptin receptor deletion from insulin- or proglucagon-expressing cells. Mol Metab. 2015;4(9):619-630.

61. Campbell JE, et al. TCF1 links GIPR signaling to the control of beta cell function and survival. Nat Med. 2016;22(1):84-90.

62. Chambers AP, et al. The Role of Pancreatic Preproglucagon in Glucose Homeostasis in Mice. Cell Metab. 2017;25(4):927-934.e3

63. Won ET, Douros JD, Hurt DA, Borski RJ. Leptin stimulates hepatic growth hormone receptor and insulin-like growth factor gene expression in a teleost fish, the hybrid striped bass. Gen Comp Endocrinol. 2016;229:84-91.

64. Afgan E, et al. The Galaxy platform for accessible, reproducible and collaborative biomedical analyses: 2016 update. Nucleic Acids Res. 2016;44(W1):W3-W10.

65. Love MI, Huber W, Anders S. Moderated estimation of fold change and dispersion for RNA-seq data with DESeq2. Genome Biol. 2014;15(12):550.

66. Marini F. pcaExplorer - Interactive exploration of Principal Components of Samples and Genes in RNA-seq data. GitHub. https://github.com/federicomarini/pcaExplorer. Accessed March 14, 2019.

67. Zhang B, Kirov S, Snoddy J. WebGestalt: an integrated system for exploring gene sets in various biological contexts. Nucleic Acids Res. 2005;33(Web Server issue):W741-W748.

68. Wikstrom JD, et al. A novel high-throughput assay for islet respiration reveals uncoupling of rodent and human islets. PLoS ONE. 2012;7(5):e33023.

69. Fisher-Wellman $\mathrm{KH}$, et al. Mitochondrial respiratory capacity and content are normal in young insulin-resistant obese humans. Diabetes. 2014;63(1):132-141.

70. Lark DS, Torres MJ, Lin CT, Ryan TE, Anderson EJ, Neufer PD. Direct real-time quantification of mitochondrial oxidative phosphorylation efficiency in permeabilized skeletal muscle myofibers. Am J Physiol, Cell Physiol. 2016;311(2):C239-C245. 